\title{
VLBI observations of nineteen GHz-peaked-spectrum radio sources at $1.6 \mathrm{GHz}$
}

\author{
X. Liu ${ }^{1}$, L. Cui ${ }^{1,2}$, W.-F. Luo ${ }^{1,2}$, W.-Z. Shi ${ }^{1,2}$, and H.-G. Song ${ }^{1}$ \\ 1 National Astronomical Observatories/Urumqi Observatory, CAS, 40-5 South Beijing Road, Urumqi 830011, PR China \\ e-mail: liux@ms.xjb.ac.cn \\ 2 Graduate University of the Chinese Academy of Sciences, Beijing 100049, PR China
}

Received 9 February 2007 / Accepted 12 April 2007

ABSTRACT

\begin{abstract}
Aims and Methods. We present the results of VLBI observations of nineteen GHz-peaked-spectrum (GPS) radio sources at 1.6 GHz. Of them, 15 sources are selected from the Parkes half-jansky (PHJ) sample (Snellen et al. 2002, MNRAS, 337, 981), and 4 others are from our previous observation list. We aimed at imaging the structure of GPS sources, searching for compact symmetric objects (CSOs) and studying the absorption for the convex radio spectra of GPS sources.

Results. We obtained total-intensity $1.6 \mathrm{GHz}$ VLBI images of 17 sources for the first time. Of them, $80 \%$ show a mini-double-lobe radio structure, indicating that they are CSOs or candidates, and their host AGNs could be edge-on to us. This result suggests that there is a high incidence of mini double-lobe sources (or CSOs) in the PHJ sample. The sources J0323+0534, J1135-0021, J1352+0232, J2058+0540, J2123-0112, and J2325-0344 are classified as CSOs with measured redshift, showing double-lobe structure with sizes of $<1 \mathrm{kpc}$. Three sources $\mathrm{J} 1057+0012$, J1600-0037, and J1753+2750 are considered as core-jet sources according to their morphologies and flux variability.
\end{abstract}

Key words. galaxies: nuclei - quasars: general - radio continuum: galaxies - galaxies: active

\section{Introduction}

The GHz-peaked-spectrum (GPS) radio sources are powerful $\left(P_{1.4 \mathrm{GHz}} \geq 10^{25} \mathrm{~W} \mathrm{~Hz}^{-1}\right)$ and compact $(\leq 1 \mathrm{kpc})$, they have convex radio spectra, and they make up a significant fraction $(\approx 10 \%)$ of the bright radio-source sample; see O'Dea (1998) for a review. In general, the presence of large-scale emission associated with GPS galaxies is rare, about a few percent in a GPS sample (Stanghellini et al. 2005). Most GPS sources appear to be truly compact and isolated.

Their small size is most likely due to their youth $\left(<10^{4}\right.$ years $)$ according to a spectral aging analysis (Murgia 2003). A couple of GPS sources are certainly young radio sources whose kinematic age from lobe proper motions has been measured and these sources are also identified as compact symmetric objects (CSOs). There is compelling evidence in favour of the youth scenario of GPS sources and CSOs, see e.g. Owsianik \& Conway (1998), Tschager et al. (2000), Polatidis \& Conway (2003), and Orienti et al. (2007). The GPS sources and CSOs are the key objects to study the early evolution of powerful radio-loud AGN. A unification scenario assumes that GPS sources evolve into compact steep spectrum sources $(1-15 \mathrm{kpc})$, which in turn evolve into classical extended radio sources $(>15 \mathrm{kpc})$, i.e. FR I/II radio sources (Fanti et al. 1995; Snellen et al. 2000; de Vries et al. 2007).

GPS galaxies are dominated by lobe/jet emission on both sides of the central engine and are thought to be relatively free of beaming effects. The GPS galaxies show very low polarization (about less than $0.5 \%$ at $5 \mathrm{GHz}$, Dallacasa 2004; Xiang et al. 2006). The low integrated polarization could be due to large Faraday depths around the radio source, which would depolarize the radio emission, implying that their host-AGNs are probably edge-on to us.

Since GPS sources live in the narrow-line region of AGN, it is likely that their low-frequency radio emission will be absorbed due to either synchrotron self-absorption or free-free absorption, giving rise to a peaked radio spectrum. Therefore, GPS sources are also suitable for studying radio absorption and scattering in AGNs.

We have carried out EVN (European VLBI Network) observations of 19 GPS sources, 15 of them from the Parkes halfjansky (PHJ) sample (Snellen et al. 2002) with declination $>-5^{\circ}$ and not observed with VLBI before. Four sources are from our previous observation list, which we observed with the EVN at 2.3/8.4 GHz and/or $5 \mathrm{GHz}$ (see Xiang et al. 2005, 2006). We aimed at imaging the GPS sources at $1.6 \mathrm{GHz}$, in order to confirm whether the GPS sources are double-lobe sources and to find CSO candidates. For the sources with observations at 2.3, 5.0 , and $8.4 \mathrm{GHz}$, the $1.6 \mathrm{GHz}$ images will provide more information on their source structure and intensity at a lower frequency, for further spectral study of the GPS sources in the future.

\section{Observations and data reduction}

The observations were carried out on 3 March 2006 at $1.65 \mathrm{GHz}$ using the MK5 recording system with a bandwidth of $32 \mathrm{MHz}$ and sample rate of $256 \mathrm{Mbps}$ in dual circular polarization. The EVN antennae in this experiment were Effelsberg, Westerbork, Jodrell, Medicina, Noto, Onsala, Torun, Hartebeesthoek, Urumqi, and Shanghai. Snapshot observations of 19 sources (Table 1) in a total of $24 \mathrm{~h}$ were made, and OQ208 
Table 1. The GPS sources.

\begin{tabular}{|c|c|c|c|c|c|c|c|c|c|c|c|c|c|c|}
\hline $\begin{array}{c}1 \\
\text { Source }\end{array}$ & 2 & $\begin{array}{c}3 \\
\text { id }\end{array}$ & $\begin{array}{c}4 \\
m_{R}\end{array}$ & $\begin{array}{l}5 \\
z\end{array}$ & $\begin{array}{c}6 \\
\mathrm{pc} / \mathrm{mas}\end{array}$ & $\begin{array}{c}7 \\
\theta \\
\text { mas }\end{array}$ & $\begin{array}{c}8 \\
L \\
\mathrm{pc}\end{array}$ & $\begin{array}{c}9 \\
S_{1.4} \\
\text { mJy }\end{array}$ & $\begin{array}{c}10 \\
S_{2.7} \\
\text { Jy }\end{array}$ & $\begin{array}{l}11 \\
\alpha_{1}\end{array}$ & $\begin{array}{l}12 \\
\alpha_{\mathrm{h}}\end{array}$ & $\begin{array}{c}13 \\
v_{\mathrm{m}} \\
\mathrm{GHz}\end{array}$ & $\begin{array}{c}14 \\
S_{\mathrm{m}} \\
\text { Jy }\end{array}$ & $\begin{array}{l}15 \\
\text { ref. }\end{array}$ \\
\hline J0210+0419 & В0208+040 & G & $18.3 \mathrm{Ks}$ & $1.5^{*}$ & 6.1 & 90 & & 948 & 0.56 & & 0.80 & 0.4 & 1.3 & 1 \\
\hline $\mathrm{J} 0323+0534$ & $4 C+05.14$ & G & 19.2 & 0.1785 & 2.7 & 180 & 490 & 2793 & 1.60 & & 0.85 & 0.4 & 7.1 & 1 \\
\hline J0433-0229 & $4 C-02.17$ & G & 19.1 & 0.530 & 5.1 & 80 & 408 & 1462 & 1.04 & & 0.52 & 0.4 & 3.0 & 1 \\
\hline J0913+1454 & B0910+151 & G & 22.9 & $0.47 *$ & 4.9 & 80 & & 881 & 0.54 & & 0.75 & 0.6 & 1.1 & 1 \\
\hline $\mathrm{J} 1057+0012$ & B1054+004 & G & 22.3 & $0.65^{*}$ & 5.5 & $80 ?$ & & 898 & 0.58 & & 0.67 & 0.4 & 1.6 & 1 \\
\hline $\mathrm{J} 1109+1043$ & B1107+109 & G & 22.6 & $0.55^{*}$ & 5.2 & 60 & & 1481 & 0.80 & & 0.94 & 0.5 & 2.4 & 1 \\
\hline J1135-0021 & $4 C-00.45$ & G & 21.9 & 0.975 & 6.0 & 120 & 720 & 1268 & 0.76 & & 0.78 & 0.4 & 2.9 & 1 \\
\hline $\mathrm{J} 1203+0414$ & B1200+045 & QSO & 18.8 & 1.221 & 6.1 & 75 & 458 & 1146 & 0.85 & & 0.45 & 0.4 & 1.4 & 1 \\
\hline $\mathrm{J} 1352+0232$ & B1349+027 & G & 20.0 & 0.607 & 5.4 & 170 & 918 & 1145 & 0.78 & & 0.58 & 0.4 & 2.0 & 1 \\
\hline $\mathrm{J} 1352+1107$ & $4 C+11.46$ & G & 21.0 & 0.891 & 5.9 & 50 & 295 & 1538 & 0.78 & & 1.03 & 0.4 & 3.6 & 1 \\
\hline J1600-0037 & В1557-004 & G & & & & 50 & & 1168 & 0.54 & & 1.17 & 1.0 & 1.2 & 1 \\
\hline $\mathrm{J} 1648+0242$ & $4 \mathrm{C}+02.43$ & G & 22.1 & 0.824 & 5.8 & & & & 0.61 & & & 0.4 & 3.4 & 1 \\
\hline $\mathrm{J} 2058+0540$ & $4 C+05.78$ & $\mathrm{G}$ & 23.4 & 1.381 & 6.1 & 160 & 970 & 1213 & 0.65 & & 0.95 & 0.4 & 3.1 & 1 \\
\hline J2123-0112 & B2121-014 & G & 23.3 & 1.158 & 6.1 & 80 & 488 & 1087 & 0.64 & -0.56 & 0.75 & 0.5 & 1.8 & 2 \\
\hline J2325-0344 & B2322-040 & $\mathrm{G}$ & 23.5 & 1.509 & 6.0 & 75 & 450 & 1224 & 0.91 & -0.42 & 0.75 & 1.4 & 1.3 & 2 \\
\hline J0917+1113 & B0914+114 & $\mathrm{EF}$ & & & & 190 & & 800 & 0.31 & -0.1 & 1.6 & 0.3 & 2.3 & 3 \\
\hline $\mathrm{J} 1753+2750$ & B1751+278 & G & 21.7 & $0.86^{*}$ & 5.9 & 50 & & 625 & 0.46 & -0.27 & 0.57 & 1.4 & 0.6 & 2 \\
\hline $\mathrm{J} 1826+2708$ & B1824+271 & G & 22.9 & & & 45 & & 332 & 0.23 & -0.39 & 0.75 & 1.0 & 0.4 & 2 \\
\hline $\mathrm{J} 2325+7917$ & B2323+790 & $\mathrm{G}$ & $19.5 \mathrm{~V}$ & & & 32 & & 1136 & & -0.3 & 0.75 & 1.4 & 1.2 & 2 \\
\hline
\end{tabular}

and DA193 were observed as calibrators. The data correlation was completed at JIVE.

The total flux densities of the sources were also measured at $5 \mathrm{GHz}$ with Urumqi $25 \mathrm{~m}$ telescope in order to find any flux variability, the values are listed in Table 2. Columns $2-4$ are flux densities at $5.0 \mathrm{GHz}$ (PKS90), $4.85 \mathrm{GHz}$ (Gregory \& Condon 1991; and Griffith et al. 1995), and $4.85 \mathrm{GHz}$ flux measured with the Urumqi $25 \mathrm{~m}$ telescope on 2007/1/24 (J1648+0242, $\mathrm{J} 2058+0540,1824+271$, and 2121-014 were not well measured due to source confusion or weak); Col. 5 is a flux variability computed from Cols. 3 and 4.

The Astronomical Image Processing System (AIPS) was used for editing, a priori calibration, fringe-fitting, selfcalibration, imaging, and model-fitting of the data.

\section{Results and comments on individual sources}

We list the basic information of the sources in Table 1: Cols. (1), (2) source names; (3) optical identification (G: galaxy, QSO: quasar, EF: empty field); (4) optical magnitude; (5) redshift (de Vries et al. 2007, those with * are a photometric estimated by Tinti et al. 2005); (6) linear scale factor $\mathrm{pc} / \mathrm{mas}\left(H_{0}=\right.$ $71 \mathrm{~km} \mathrm{~s}^{-1} \mathrm{Mpc}^{-1}$ and $q_{0}=0.5$ have been assumed); (7) maximum angular size from the observation; (8) maximum linear size; (9) $1.4 \mathrm{GHz}$ flux density from the NVSS; (10) $2.7 \mathrm{GHz}$ flux density from Snellen sample and the NED; (11) low frequency spectral index; (12) higher frequency spectral index (computed from Cols. 9 and 10), $S \propto v^{-\alpha}$; (13) turnover frequency; (14) peak flux density; (15) references for the spectral information, 1 Snellen et al. (2002), 2 de Vries et al. (1997), 3 Stanghellini et al. (1998). The parameters derived from the VLBI images are in Table 3: columns give (1) source name and possible classification (CSOc: CSO candidate, cj: core-jet); (2) total cleaned flux density of image at $1.6 \mathrm{GHz}$; (3) component identification labled to Xiang et al. 2002, 2005, 2006; (4), (5) peak and integral intensity of a fitted Gaussian component at $1.6 \mathrm{GHz}$ in the AIPS task JMFIT; (6), (7) major/minor axes and position angle of component at $1.6 \mathrm{GHz} ;(8),(9)$ distance and position angle relative to
Table 2. Source total flux density at $5 \mathrm{GHz}$ and possible variability.

\begin{tabular}{ccccc}
\hline \hline Source & $\begin{array}{c}S_{5.0} \\
\mathrm{mJy}\end{array}$ & $\begin{array}{c}S_{4.85} \\
\mathrm{mJy}\end{array}$ & $\begin{array}{c}S_{4.85 \mathrm{Ur}} \\
\mathrm{mJy}\end{array}$ & $\begin{array}{c}\delta S_{4.85} \\
\%\end{array}$ \\
\hline $\mathrm{J} 0210+0419$ & 300 & $298 \pm 19$ & $302 \pm 10$ & $1.3 \pm 3.1$ \\
$\mathrm{~J} 0323+0534$ & 830 & $819 \pm 44$ & $868 \pm 9$ & $6.0 \pm 4.6$ \\
$\mathrm{~J} 0433-0229$ & 640 & $640 \pm 35$ & $637 \pm 14$ & $-0.5 \pm 3.3$ \\
$\mathrm{~J} 0913+1454$ & 300 & $315 \pm 43$ & $297 \pm 8$ & $-5.7 \pm 10.3$ \\
$\mathrm{~J} 1057+0012$ & 370 & $396 \pm 23$ & $351 \pm 6$ & $-11.4 \pm 3.6$ \\
$\mathrm{~J} 1109+1043$ & 400 & $408 \pm 56$ & $428 \pm 8$ & $4.9 \pm 12.4$ \\
$\mathrm{~J} 1135-0021$ & 440 & $446 \pm 25$ & $427 \pm 8$ & $-4.3 \pm 3.6$ \\
$\mathrm{~J} 1203+0414$ & 520 & $640 \pm 35$ & $611 \pm 7$ & $-4.5 \pm 4.1$ \\
$\mathrm{~J} 1352+0232$ & 470 & & $469 \pm 7$ & \\
$\mathrm{~J} 1352+1107$ & 410 & $447 \pm 62$ & $418 \pm 5$ & $-6.5 \pm 11.9$ \\
$\mathrm{~J} 1600-0037$ & 180 & $187 \pm 14$ & $212 \pm 3$ & $13.4 \pm 6.9$ \\
$\mathrm{~J} 1648+0242$ & 260 & $337 \pm 20$ & & \\
$\mathrm{~J} 2058+0540$ & 340 & $356 \pm 21$ & & \\
B2121-014 & 320 & $345 \pm 21$ & & \\
$\mathrm{~B} 2322-040$ & 500 & $524 \pm 29$ & $545 \pm 20$ & $4.0 \pm 1.9$ \\
$\mathrm{~B} 0914+114$ & 140 & $134 \pm 19$ & $140 \pm 1$ & $4.5 \pm 14.1$ \\
$\mathrm{~B} 1751+278$ & & $298 \pm 39$ & $292 \pm 6$ & $-2.0 \pm 10.8$ \\
$\mathrm{~B} 1824+271$ & & $122 \pm 17$ & & \\
$\mathrm{~B} 2323+790$ & & & $491 \pm 7$ & \\
OQ208 & & $2421 \pm 217$ & $2514 \pm 12$ & $3.8 \pm 8.8$ \\
\hline
\end{tabular}

the first component; (10) brightness temperature of component. We comment here on the results of each source and give a short discussion. We use $S \propto v^{-\alpha}$ to define the spectral index. Optical information and redshifts of the GPS sources in the PHJ sample are given by de Vries et al. (2007), as listed in Table 1.

\subsection{J0210+0419 (PKS B0208+040)}

The $1.6 \mathrm{GHz}$ VLBI image (Fig. 1) is the first VLBI image of the source. It shows a double-lobe structure and is most likely a CSO. Optical observations did not result in an identification with 
Table 3. The component parameters of the VLBI images at $1.6 \mathrm{GHz}$.

\begin{tabular}{|c|c|c|c|c|c|c|c|c|c|}
\hline $\begin{array}{c}1 \\
\text { Name } \\
\text { class }\end{array}$ & $\begin{array}{c}2 \\
S_{\text {vlbi }} \\
\text { mJy }\end{array}$ & $\begin{array}{c}3 \\
\text { Comp }\end{array}$ & $\begin{array}{c}4 \\
\text { Sp } \\
\text { mJy }\end{array}$ & $\begin{array}{c}5 \\
\text { Sint } \\
\text { mJy }\end{array}$ & $\begin{array}{c}6 \\
\theta_{1} \times \theta_{2} \\
\operatorname{mas} \times \text { mas }\end{array}$ & $\begin{array}{c}7 \\
\text { PA } \\
0\end{array}$ & $\begin{array}{c}8 \\
d \\
\text { mas }\end{array}$ & $\begin{array}{c}9 \\
\mathrm{PA} \\
\circ\end{array}$ & $\begin{array}{c}10 \\
T_{\mathrm{b}} \\
10^{8} \mathrm{~K}^{\circ}\end{array}$ \\
\hline J0210+0419 & 715 & A & 290 & 375 & $5.7 \times 2.9$ & 169 & 0 & & 11.9 \\
\hline CSOc & & B & 118 & 205 & $10 \times 3.6$ & 175 & $68.2 \pm 0.1$ & $-153.2 \pm 0.1$ & 2.2 \\
\hline $\mathrm{J} 0323+0534$ & 1497 & A & 536 & 1270 & $32.6 \times 11.1$ & 70 & 0 & & 0.5 \\
\hline CSO & & B & 117 & 548 & $57.5 \times 21.7$ & 18 & $122.7 \pm 2.9$ & $-167.2 \pm 0.4$ & 0.1 \\
\hline J0433-0229 & 1095 & A & 407 & 1045 & $14.5 \times 4.7$ & 171 & 0 & & 2.4 \\
\hline CSOc/cj & & B & 49 & 111 & $9.9 \times 5.5$ & 88 & $53.0 \pm 0.3$ & $164.4 \pm 0.2$ & 0.3 \\
\hline J0913+1454 & 796 & A & 211 & 501 & $8.2 \times 4.9$ & 65 & 0 & & 2.1 \\
\hline $\mathrm{CSOc}$ & & B & 55 & 157 & $8.9 \times 6.2$ & 80 & $56.8 \pm 0.1$ & $73.3 \pm 0.1$ & 0.4 \\
\hline $\mathrm{J} 1057+0012$ & 810 & A & 381 & 550 & $3.6 \times 2.7$ & 6.6 & 0 & & 17.5 \\
\hline cj & & B & 37 & 58 & $6.2 \times 2.1$ & 9.7 & $9.7 \pm 0.2$ & $114.8 \pm 0.9$ & 1.3 \\
\hline $\mathrm{J} 1109+1043$ & 1370 & A & 420 & 984 & $5.7 \times 4.7$ & 110 & 0 & & 6.6 \\
\hline CSOc & & B & 143 & 311 & $5.4 \times 4.2$ & 91 & $46.3 \pm 0.1$ & $104.4 \pm 0.1$ & 2.6 \\
\hline J1135-0021 & 1025 & A & 279 & 454 & $6.6 \times 2.7$ & 142 & 0 & & 4.9 \\
\hline CSO & & B & 143 & 271 & $8.6 \times 3.0$ & 153 & $85.8 \pm 0.1$ & $164.4 \pm 0.1$ & 1.7 \\
\hline J1203+0414 & 1029 & A & 571 & 850 & $4.7 \times 2.9$ & 107 & 0 & & 25.1 \\
\hline \multirow[t]{2}{*}{$\mathrm{CSOc}$} & & B & 51 & 81 & $5.0 \times 3.8$ & 78 & $18.1 \pm 0.2$ & $103.0 \pm 0.4$ & 1.6 \\
\hline & & $\mathrm{C}$ & 31 & 35 & $6 \times 6$ & 0 & $58.2 \pm 0.2$ & $104.2 \pm 0.2$ & 1.9 \\
\hline $\mathrm{J} 1352+0232$ & 885 & A & 173 & 480 & $7.4 \times 4.5$ & 53 & 0 & & 2.1 \\
\hline CSO & & B & 30 & 120 & $8.9 \times 6.3$ & 110 & $165.7 \pm 0.3$ & $-111.9 \pm 0.1$ & 0.2 \\
\hline $\mathrm{J} 1352+1107$ & 896 & A & 198 & 395 & $8.1 \times 5.4$ & 11.7 & 0 & & 2.0 \\
\hline CSOc/cj & & B & 106 & 202 & $6.8 \times 6.3$ & 10 & $3.5 \pm 0.1$ & $53.1 \pm 0.1$ & 1.1 \\
\hline J1600-0037 & 936 & A & 394 & 607 & $5.4 \times 4.1$ & 157 & 0 & & \\
\hline cj & & B & 125 & 255 & $7.9 \times 5.0$ & 86 & $24.7 \pm 0.1$ & $83.5 \pm 0.1$ & \\
\hline J2058+0540 & 914 & A & 356 & 513 & $7.6 \times 3.7$ & 163 & 0 & & 8.2 \\
\hline CSO & & B & 182 & 403 & $12.6 \times 4.5$ & 128 & $127.2 \pm 0.1$ & $172.1 \pm 0.1$ & 2.3 \\
\hline B2121-014 & 976 & A & 363 & 594 & $5.1 \times 3.9$ & 123 & 0 & & 10.4 \\
\hline CSO & & $\mathrm{C}$ & 191 & 415 & $7.4 \times 5.0$ & 126 & $59.4 \pm 0.1$ & $85.4 \pm 0.1$ & 2.9 \\
\hline B2322-040 & 965 & A & 229 & 509 & $14.6 \times 3.3$ & 161 & 0 & & 3.6 \\
\hline \multirow[t]{2}{*}{ CSO } & & B & 74 & 160 & $11.7 \times 5.3$ & 162 & $40.6 \pm 0.2$ & $171.3 \pm 0.1$ & 0.8 \\
\hline & & $\mathrm{C}$ & 65 & 196 & $19.3 \times 4.7$ & 1 & $22.0 \pm 0.4$ & $171.1 \pm 0.2$ & 0.5 \\
\hline B0914+114 & 578 & A & 37 & 50 & $4.9 \times 2.7$ & 16 & 0 & & \\
\hline \multirow[t]{3}{*}{ CSO } & & B & 29 & 65 & $9.1 \times 4.3$ & 66 & $45.6 \pm 0.1$ & $80.1 \pm 0.1$ & \\
\hline & & $\mathrm{C}$ & 242 & 360 & $4.2 \times 3.9$ & 90 & $84.2 \pm 0.1$ & $81.6 \pm 0.1$ & \\
\hline & & E & 28 & 40 & $4.4 \times 3.4$ & 63 & $86.1 \pm 0.1$ & $-95.9 \pm 0.1$ & \\
\hline B $1751+278$ & 596 & A & 400 & 522 & $4.8 \times 2.9$ & 71 & 0 & & 14.5 \\
\hline \multirow[t]{3}{*}{ cj } & & B & 26 & 37 & $8 \times 3$ & 15 & $20.5 \pm 0.1$ & $-128.6 \pm 0.2$ & 0.5 \\
\hline & & $\mathrm{C}$ & 12.4 & 21 & $7 \times 5$ & 59 & $26.6 \pm 0.3$ & $-119.8 \pm 0.3$ & 0.2 \\
\hline & & D & 8 & 20 & $19.5 \times 3.6$ & 176 & $41.4 \pm 0.4$ & $-104.4 \pm 0.8$ & 0.1 \\
\hline B1824+271 & 296 & A & 145 & 174 & $3.9 \times 2.1$ & 164 & 0 & & \\
\hline CSO & & B & 42 & 69 & $6.1 \times 4.3$ & 137 & $21.8 \pm 0.1$ & $-83.4 \pm 0.1$ & \\
\hline B2323+790 & 900 & A & 439 & 621 & $5.8 \times 2.1$ & 159 & 0 & & \\
\hline $\mathrm{CSOc}$ & & $B+C$ & 107 & 155 & $7.9 \times 3.1$ & 118 & $19.2 \pm 0.1$ & $-71.1 \pm 0.1$ & \\
\hline
\end{tabular}

a lower limit of $m_{R}>24.1$, but it is identified with a magnitude of $K \mathrm{~s}=18.3$ (de Vries et al. 2007).

\section{2. $J 0323+0534(4 C+05.14)$}

The 1.6 GHz VLBI image (Fig. 2) is the first VLBI image of the source, and it exhibits a strong diffuse component and a weak extended component in the south. Both are probably lobe emission. About $38 \%$ total flux density (estimated from Table 1) is resolved out in the VLBI image, due to the diffuse components. For its size of $490 \mathrm{pc}$, the source can be a CSO.

\subsection{J0433-0229 (4C-02.17)}

The $1.6 \mathrm{GHz}$ VLBI image (Fig. 3) is the first VLBI image of the source, and the main component is diffuse and extended in the north-south direction and a possible weak component in the south. About $18 \%$ total flux density (estimated from Table 1) is resolved out in the VLBI image. Either a core-jet or a CSO classification is possible for the source.

\subsection{J0913+1454 (PKS B0910+151)}

The 1.6 GHz VLBI image (Fig. 4) is the first VLBI image of the source. It shows a double structure, and both components are further resolved. There is probably a hotspot imbedded in the bright one. We consider it a CSO candidate.

\subsection{J1057+0012 (PKS B1054+004)}

The $1.6 \mathrm{GHz}$ VLBI image (Fig. 5) is the first VLBI image of the source. There is a bright compact component followed by a secondary component and a series of possibly weak components in the east, indicating this is a core-jet source. A flux variability 


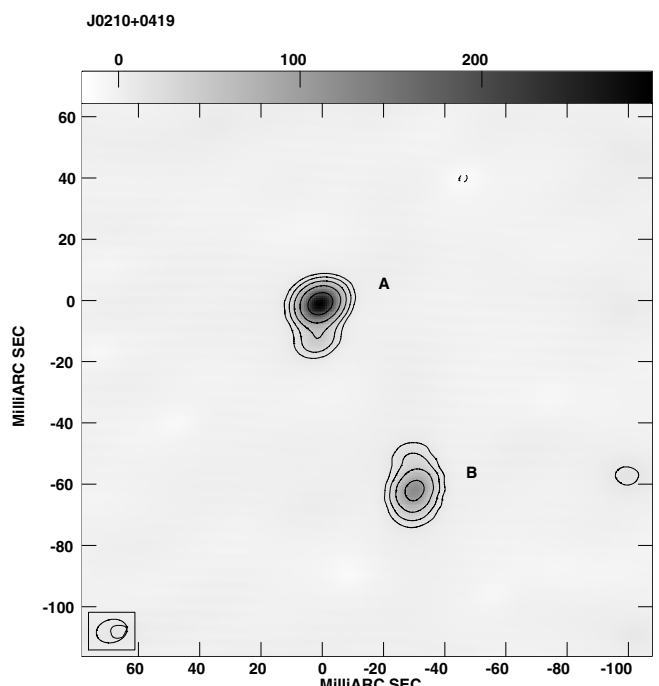

Fig. 1. J0210+0419 at $1.65 \mathrm{GHz}$. The restoring beam is $10.2 \times$ 7.5 mas with $\mathrm{PA}-75.5^{\circ}$, the peak is $293 \mathrm{mJy} /$ beam, the contours are $12 \mathrm{mJy} /$ beam times levels $-1,1,2,4,8,16,32,64,100,200,400,800$, and the same levels are used in the following images. The grey scale unit is mJy.

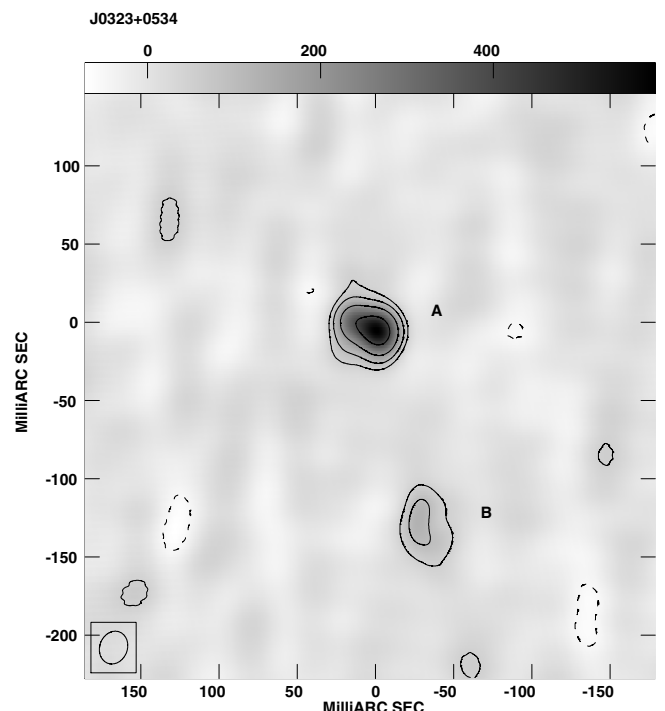

Fig. 2. J0323+0534 at $1.65 \mathrm{GHz}$. The restoring beam is $21.3 \times 17.7$ mas with $\mathrm{PA}-20.2^{\circ}$, the peak is $586 \mathrm{mJy} /$ beam, and the first contour is $50 \mathrm{mJy} /$ beam.

of $(-11.4 \pm 3.6) \%$ over 15 years at $5 \mathrm{GHz}$, as reported in Table 2, is consistent with the core-jet classification.

\subsection{J1109+1043 (PKS B1107+109)}

The $1.6 \mathrm{GHz}$ VLBI image (Fig. 6) is the first VLBI image of the source. It is a double structure and can be a CSO candidate. The total flux density (1270 mJy estimated from Table 1 ) is completely restored in the VLBI image ( $1370 \mathrm{mJy}$, increased by $8 \%)$. There is also an indication of total flux increasing $(4.9 \pm 12.4) \%$ at $5 \mathrm{GHz}$ in Table 2 but with a large error.

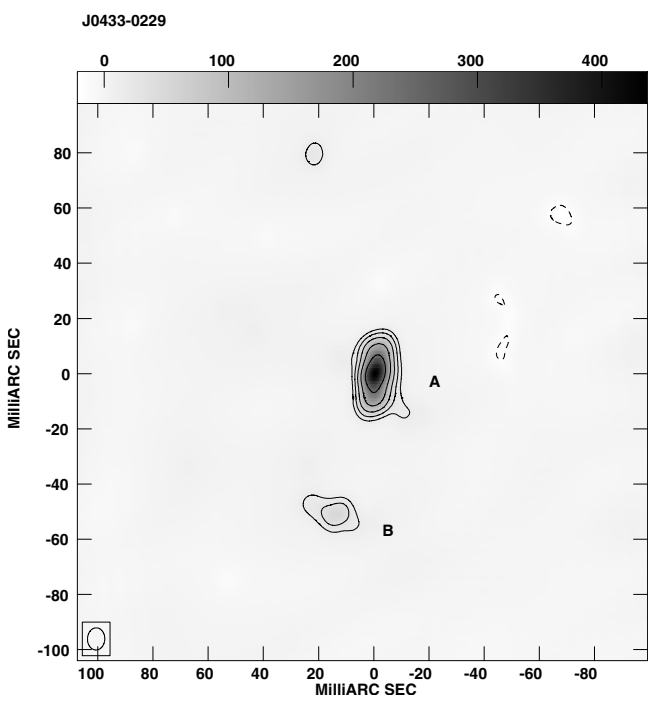

Fig. 3. J0433-0229 at $1.65 \mathrm{GHz}$. The restoring beam is $8.0 \times 6.3$ mas with $\mathrm{PA}-1.7^{\circ}$, the peak is $435 \mathrm{mJy} / \mathrm{beam}$, and the first contour is $15 \mathrm{mJy} /$ beam

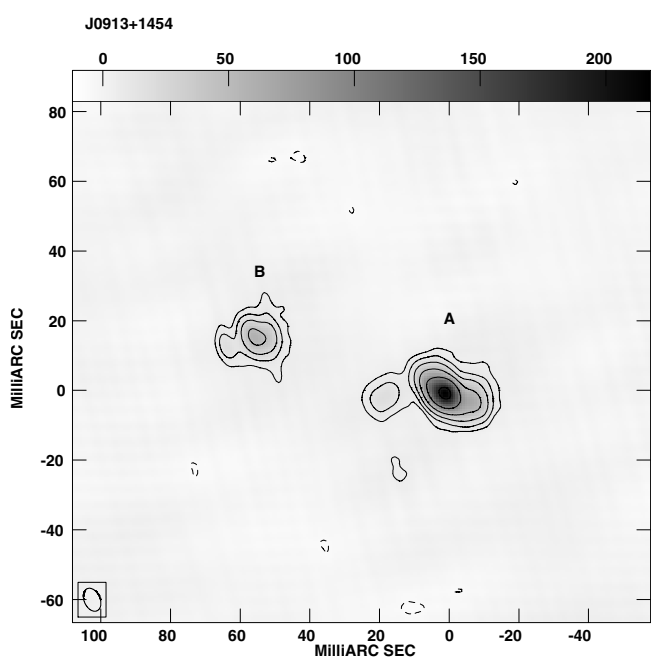

Fig. 4. J0913+1454 at $1.65 \mathrm{GHz}$. The restoring beam is $6.9 \times 4.6$ mas with PA $24.4^{\circ}$, the peak is $217 \mathrm{mJy} / \mathrm{beam}$, and the first contour is $6 \mathrm{mJy} / \mathrm{beam}$.

\subsection{J1135-0021 (4C-00.45)}

The $1.6 \mathrm{GHz}$ VLBI image (Fig. 7) is the first VLBI image of the source. It shows a double-lobe structure, and with its size of $720 \mathrm{pc}$, we classify the source as a CSO.

\subsection{J1203+0414 (PKS B1200+045)}

The 1.6 GHz VLBI image (Fig. 8) is the first VLBI image of the source. The triple structure may consist of a core and two-sided emission, or a one-sided core-jet source. The quasar, as newly identified by de Vries et al. (2007), is possibly a core-jet one, but still we keep the source as a CSO candidate.

\subsection{J1352+0232 (PKS B1349+027)}

The 1.6 GHz VLBI image (Fig. 9) is the first VLBI image of the source. It shows a double-lobe like structure, and we consider it as a CSO because of its size of 918 pc. 


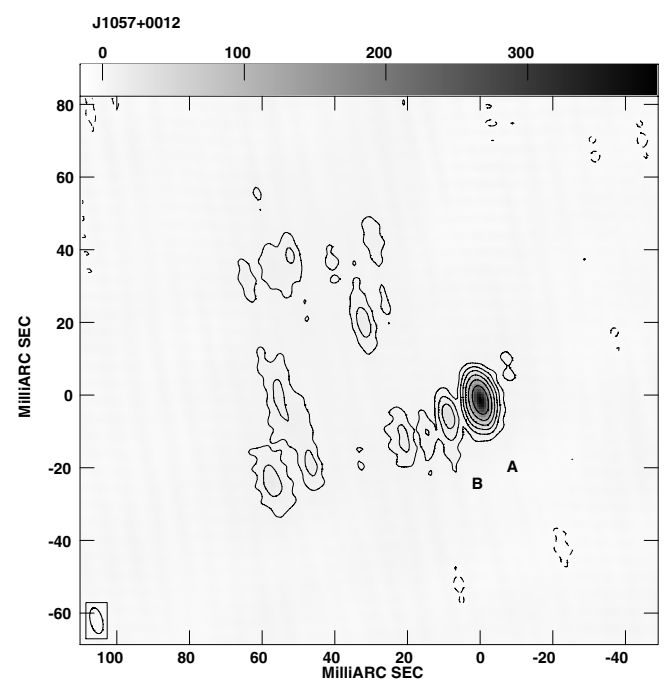

Fig. 5. J1057+0012 at $1.65 \mathrm{GHz}$. The restoring beam is $7.3 \times 3.3$ mas with PA $13.4^{\circ}$, the peak is $390 \mathrm{mJy} / \mathrm{beam}$, and the first contour is $6 \mathrm{mJy} /$ beam.

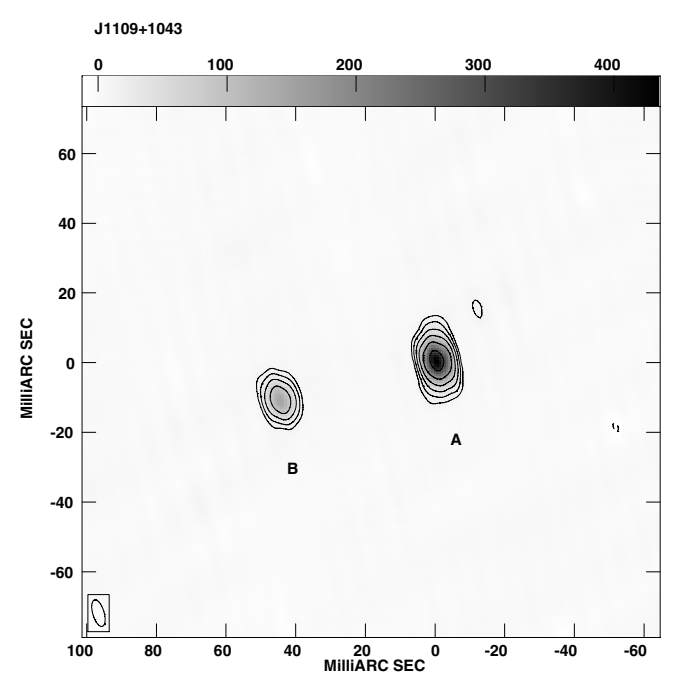

Fig. 6. J1109+1043 at $1.65 \mathrm{GHz}$. The restoring beam is $7.9 \times 3.3$ mas with PA $16.5^{\circ}$, the peak is $434 \mathrm{mJy} / \mathrm{beam}$, and the first contour is $10 \mathrm{mJy} / \mathrm{beam}$.

\subsection{0. $J 1352+1107(4 C+11.46)$}

The 1.6 GHz VLBI image (Fig. 10) is the first VLBI image of the source. It appears to have a compact double structure or a corejet, and seems diffuse emission around the source. About $30 \%$ total flux density (estimated from Table 1) is resolved out in the VLBI image. Either a corejet or a compact double classification is possible.

\subsection{J1600-0037 (PKS B1557-004)}

The 1.6 GHz VLBI image (Fig. 11) is the first VLBI image of the source, and it has an overall double structure, while the eastern component has some extension in the west-east direction. A flux variability of $(13 \pm 6.9) \%$ at $5 \mathrm{GHz}$ in Table 2 may suggest this is a core-jet source.

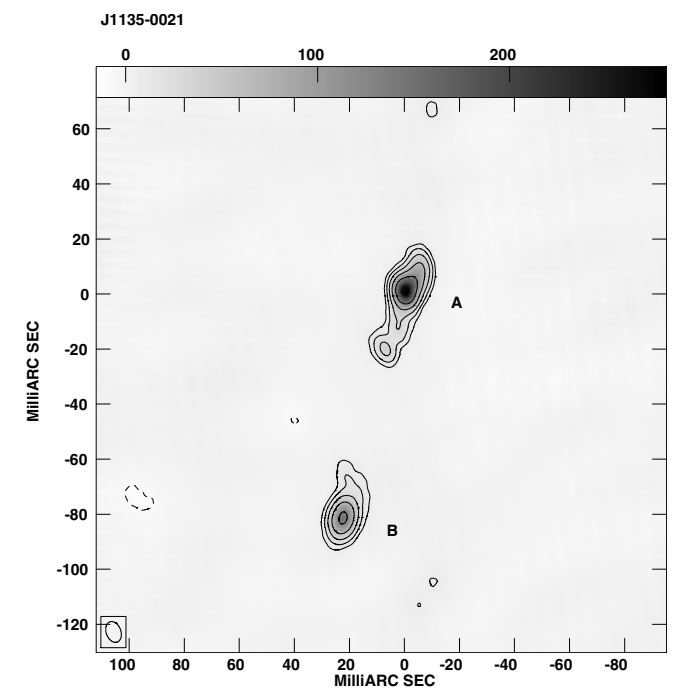

Fig. 7. J1135-0021 at $1.65 \mathrm{GHz}$. The restoring beam is $7.8 \times 5.3$ mas with PA $20.4^{\circ}$, the peak is $281 \mathrm{mJy} / \mathrm{beam}$, and the first contour is $8 \mathrm{mJy} / \mathrm{beam}$.

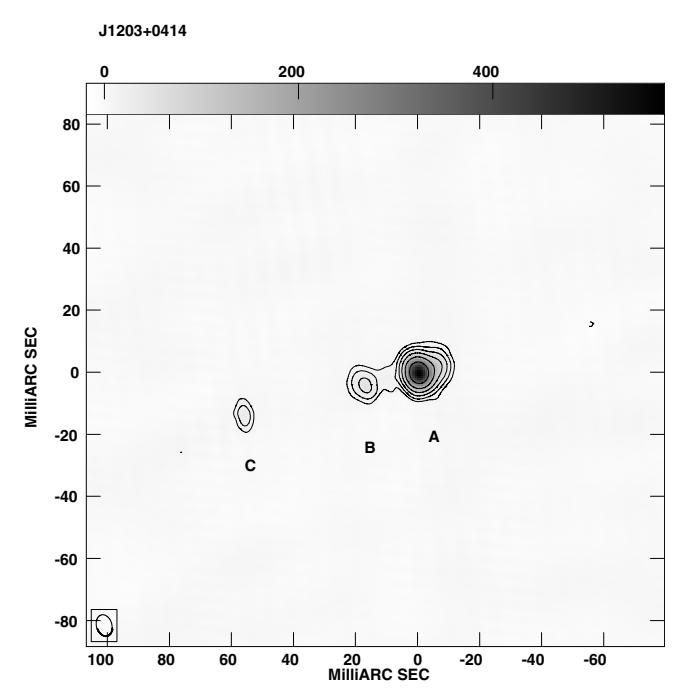

Fig. 8. J1203+0414 at $1.65 \mathrm{GHz}$. The restoring beam is $7.0 \times 4.9$ mas with PA $15.2^{\circ}$, the peak is $575 \mathrm{mJy} / \mathrm{beam}$, and the first contour is $10 \mathrm{mJy} /$ beam.

\subsection{2. $J 1648+0242(4 C+02.43)$}

The GPS source is not detected with VLBI. It is an NVSS double-lobe source, and totally resolved out in the VLBI observation.

\subsection{3. $J 2058+0540(4 C+05.78)$}

The 1.6 GHz VLBI image (Fig. 12) is the first VLBI image of the source. It shows a double-lobe source, and because of its size of $970 \mathrm{pc}$, we suggest this is a CSO.

\subsection{PKS B2121-014}

The 1.6 GHz VLBI image (Fig. 13) shows a double-lobe structure, and it is similar to that at 2.3 and $5 \mathrm{GHz}$ (Xiang et al. 2005, 2006), except that a weak jet-like emission "B" appearing at 2.3 and $5 \mathrm{GHz}$ is missing, probably due to absorption at the lower 


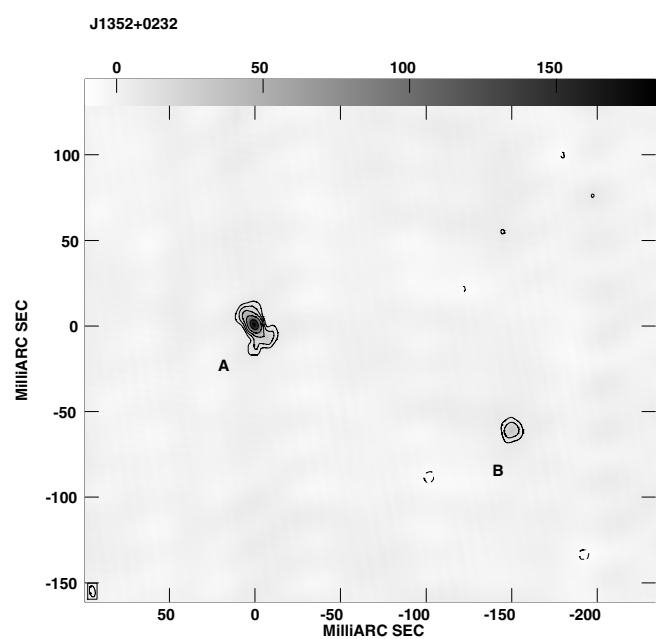

Fig. 9. J1352+0232 at $1.65 \mathrm{GHz}$. The restoring beam is $6.7 \times 3.2 \mathrm{mas}$ with PA $9.9^{\circ}$, the peak is $183 \mathrm{mJy} /$ beam, and the first contour is $8 \mathrm{mJy} /$ beam.

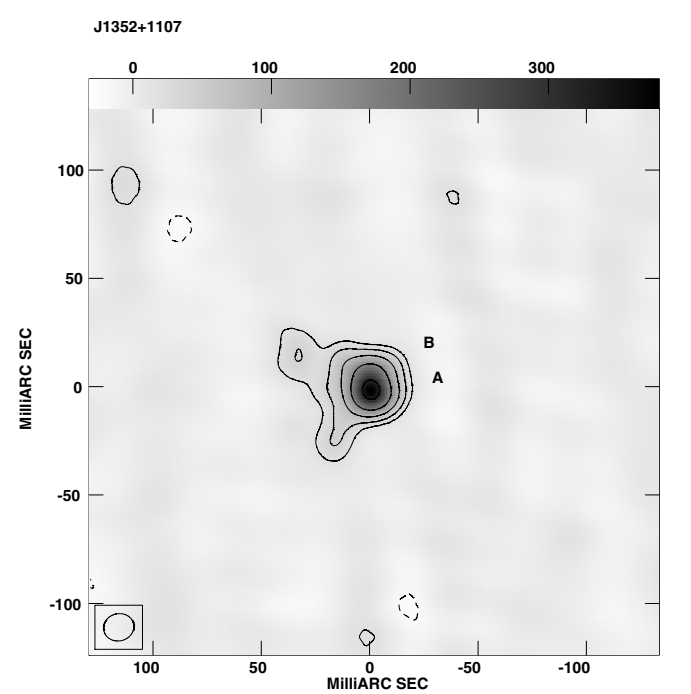

Fig. 10. J1352+1107 at $1.65 \mathrm{GHz}$. The restoring beam is $14.0 \times 12.5$ mas with PA $-75.3^{\circ}$, the peak is $379 \mathrm{mJy} /$ beam, and the first contour is $20 \mathrm{mJy} /$ beam.

frequency $1.6 \mathrm{GHz}$. The source is a CSO for the source size of 488 pc.

\subsection{PKS B2322-040}

The 1.6 GHz VLBI image (Fig. 14) exposes a central emission region between the two lobes "A" and "B", which is probably a core embedded in the central region. The "core" emission is not detected at higher frequencies (Xiang et al. 2005, 2006), but it emerges at $1.6 \mathrm{GHz}$ near the peak frequency $(1.4 \mathrm{GHz})$ of the GPS source. There is a flux increase of $(4.0 \pm 1.9) \%$ over 15 years at $5 \mathrm{GHz}$ (Table 2), suggesting that the core is currently active. The source can be a CSO from its size of $450 \mathrm{pc}$.

\subsection{PKS B0914+114}

The 1.6 GHz VLBI image (Fig. 15) exhibits a core "A", jet feature "B", and two lobes "C" and "E". The western one "E" emerges at this frequency. Labiano et al. (2007) have identified

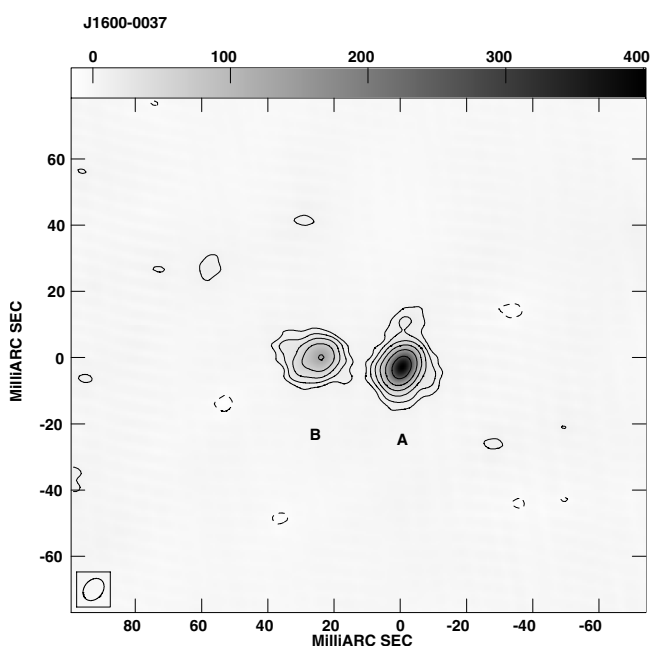

Fig. 11. J1600-0037 at $1.65 \mathrm{GHz}$. The restoring beam is $7.4 \times 5.5$ mas with PA $-39.8^{\circ}$, the peak is $400 \mathrm{mJy} /$ beam, and the first contour is $8 \mathrm{mJy} /$ beam.

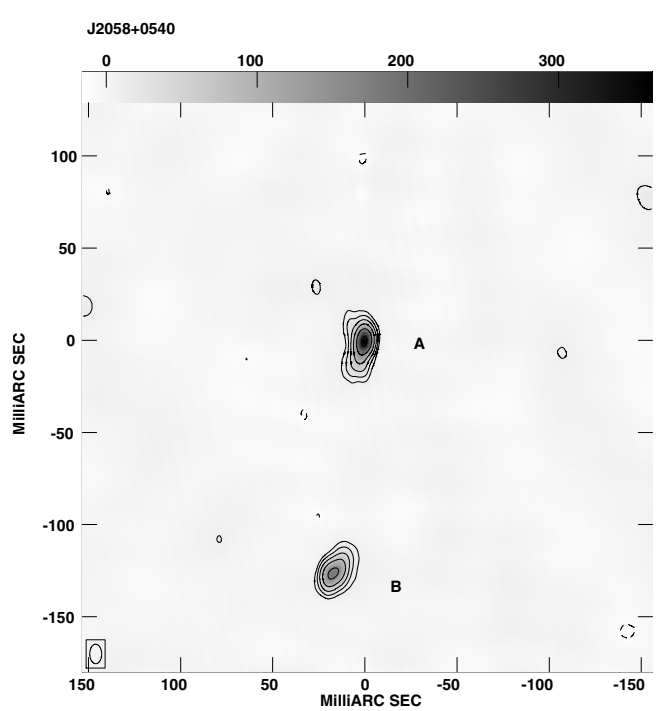

Fig. 12. J2058+0540 at $1.65 \mathrm{GHz}$. The restoring beam is $10.6 \times 6.5$ mas with PA $0.7^{\circ}$, the peak is $362 \mathrm{mJy} /$ beam, and the first contour is $10 \mathrm{mJy} / \mathrm{beam}$.

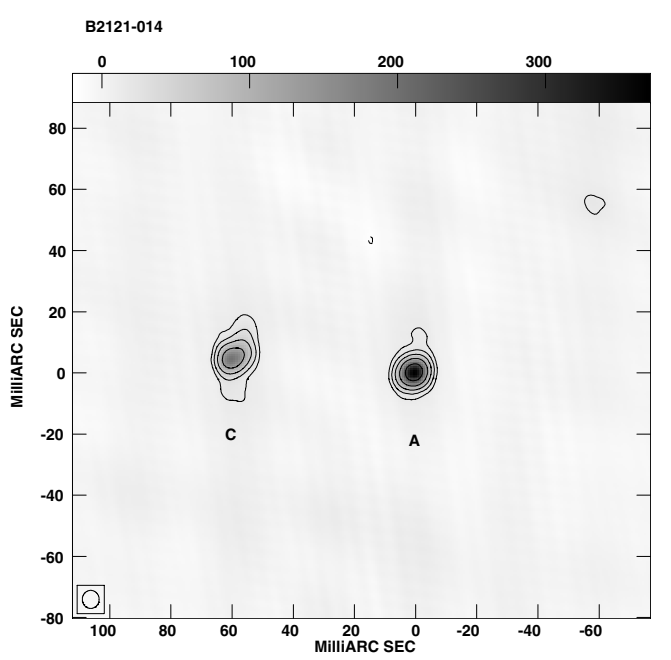

Fig. 13. $2121-014$ at $1.65 \mathrm{GHz}$. The restoring beam is $5.9 \times 5.5$ mas with PA $-1.4^{\circ}$, the peak is $370 \mathrm{mJy} / \mathrm{beam}$, and the first contour is $15 \mathrm{mJy} /$ beam. 


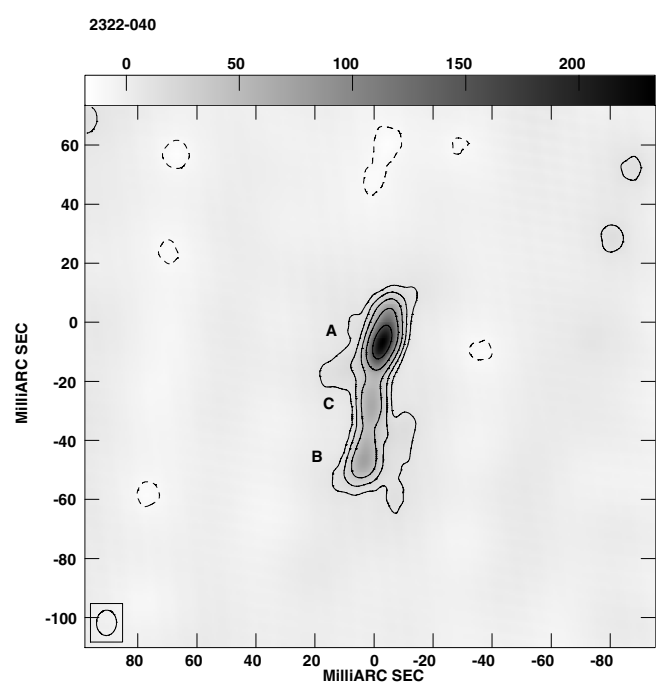

Fig. 14. $2322-040$ at $1.65 \mathrm{GHz}$. The restoring beam is $8.6 \times 6.8$ mas with PA $-2.1^{\circ}$, the peak is $233 \mathrm{mJy} / \mathrm{beam}$, and the first contour is $10 \mathrm{mJy} / \mathrm{beam}$.

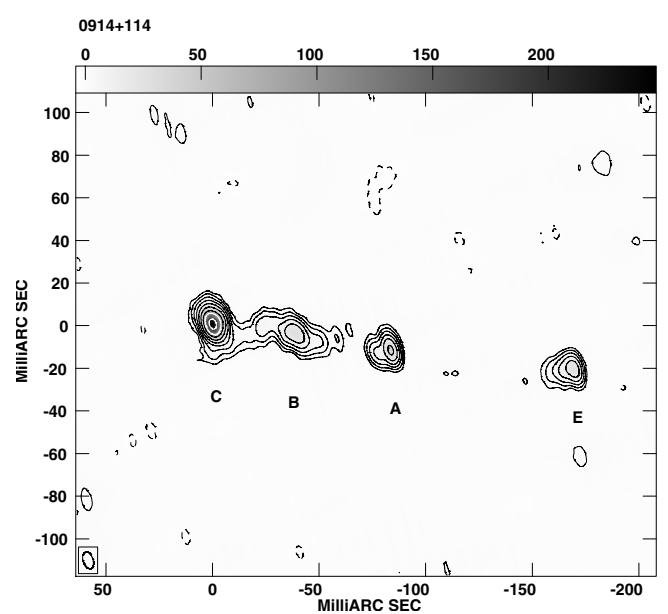

Fig. 15. $0914+114$ at $1.65 \mathrm{GHz}$. The restoring beam is $8.3 \times 4.7$ mas with PA $16.8^{\circ}$, the peak is $246 \mathrm{mJy} / \mathrm{beam}$, and the first contour is $1 \mathrm{mJy} / \mathrm{beam}$.

an empty field $\left(>25 m_{R}\right)$ at the FIRST position of the source and conclude that the previously identified, nearby disk galaxy (a redshift of 0.178) is not the host to this radio source 0914+114. For the typical compact, symmetric structure, we consider the source is a CSO.

\subsection{7. $1751+278$ (MG2 J175301+2750)}

The $1.6 \mathrm{GHz}$ structure (Fig. 16) is similar to what we got before at $1.6 \mathrm{GHz}$ (Xiang et al. 2002), which confirms that there is jet-like emission " $C$ " and "D" associated with the southern component " $\mathrm{B}$ ", indicating this is a core-jet source.

\subsection{B2 $1824+271$}

The 1.6 GHz VLBI image (Fig. 17) exposes a symmetric double structure and jet-like emission associated with the two lobes, confirming this is a CSO as we suggested (Xiang et al. 2006).

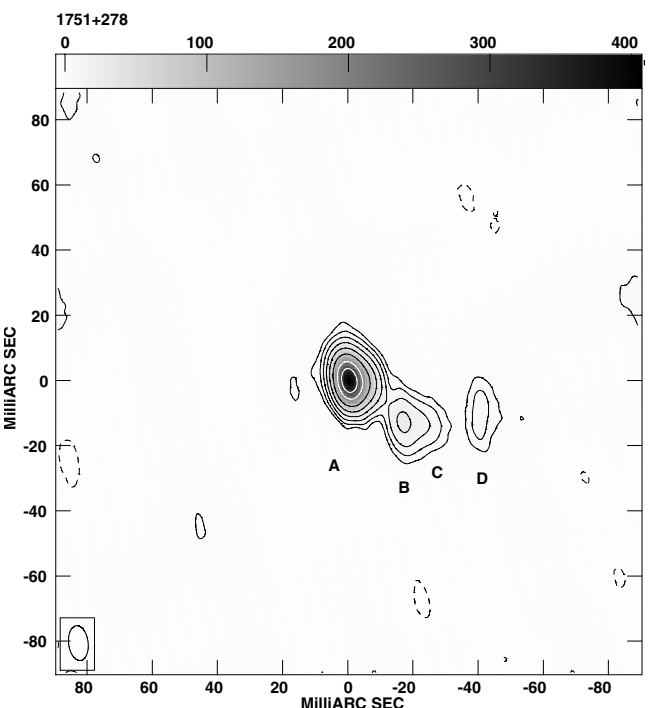

Fig. 16. $1751+278$ at $1.65 \mathrm{GHz}$. The restoring beam is $10.7 \times 6.0$ mas with PA $7.0^{\circ}$, the peak is $406 \mathrm{mJy} /$ beam, and the first contour is $3 \mathrm{mJy} /$ beam.

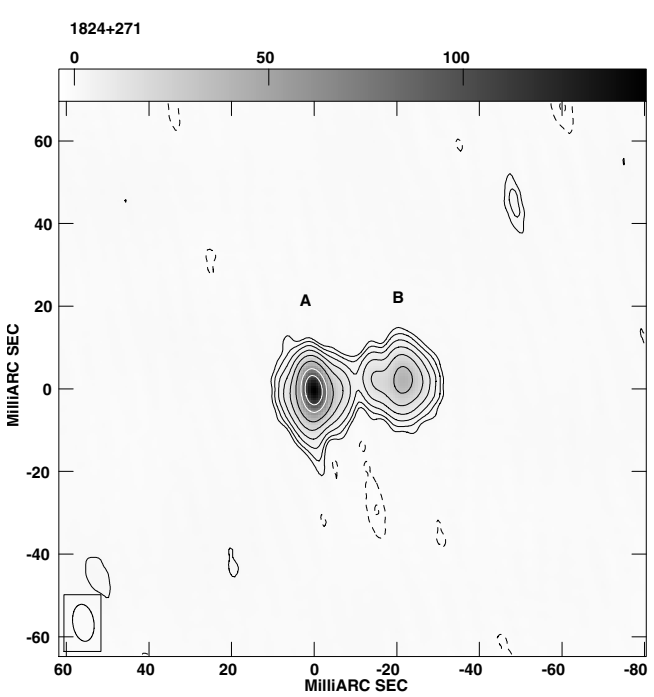

Fig. 17. $1824+271$ at $1.65 \mathrm{GHz}$. The restoring beam is $9.0 \times 5.1$ mas with PA $8.1^{\circ}$, the peak is $146 \mathrm{mJy} / \mathrm{beam}$, and the first contour is $1 \mathrm{mJy} / \mathrm{beam}$.

\subsection{9. [WB92] 2323+790}

The $1.6 \mathrm{GHz}$ image (Fig. 18) shows a central component "A" and a weak one "B+C" in the northwest, and the components "A" and "B+C" show steep spectra between $1.6 \mathrm{GHz}$ and $5 \mathrm{GHz}$ (Xiang et al. 2006). The source can be a CSO candidate.

\section{Discussion}

In the sample (Table 1), J1648+0242 is an NVSS double source and is not detected in this VLBI observation; all others are point-like in the NVSS images, indicating that GPS sources are compact. Except for four sources $(\mathrm{J} 1057+0012$, J1352+1107, $\mathrm{J} 1600-0037$, and $1751+278), 14$ out of 18 sources exhibit a double or triple VLBI structure and can be CSOs or CSO candidates, though some of them have no measured redshift. The sources with redshift show double or triple structure with sizes $<1 \mathrm{kpc}$, suggesting these GPS sources are certainly compact and likely CSOs. 


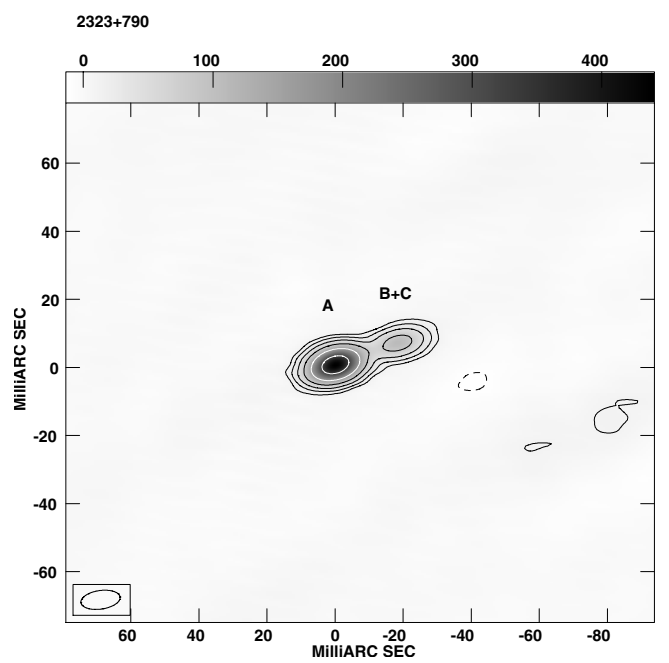

Fig. 18. $2323+790$ at $1.65 \mathrm{GHz}$. The restoring beam is $11.6 \times 5.4$ mas with PA $-82^{\circ}$, the peak is $438 \mathrm{mJy} / \mathrm{beam}$, and the first contour is $10 \mathrm{mJy} /$ beam.

The mini double-lobe sources or CSOs could be more stable in flux density than other types of compact sources. We measured the flux densities for the sources (Table 2) at $4.85 \mathrm{GHz}$ and compared them with the values observed 15 years ago. We find that 12 among the 14 GPS sources are probably stable in flux ( $1 \sigma$ level), and two sources (J1057+0012 and J1600-0037) show about $10 \%$ variability on the $3 \sigma$ and $2 \sigma$ levels respectively. The flux variability on J1057+0012 and J1600-0037 is consistent with their core-jet classification. "Core-jet" sources are defined to show a one-sided jet, and the jet is often closely pointing to us (from a pole-on AGN). It is hard to estimate the real source size due to Doppler boosting, hence the "core-jet" sources might not be young radio sources even if they appear to be compact in some cases.

In addition, some sources are resolved out in our VLBI image by more than $10 \%$ of the total flux estimated from Table 1, probably due to diffuse emission associated with lobes and tail/jet emission. They are J0210+0419 (-14\%), $\mathrm{J} 0323+0534 \quad(-38 \%), \quad \mathrm{J} 0433-0229 \quad(-18 \%), \quad \mathrm{J} 1352+0232$ $(-15 \%), \mathrm{J} 1135+1107(-31 \%), \mathrm{J} 2058+0540$ (-12\%), 2322-040 $(-15 \%)$, and $\mathrm{J} 1648+0242$ is completely resolved out. The VLBI flux densities of the other nine sources at $1.6 \mathrm{GHz}$ are consistent with the estimated total flux densities within an error of $10 \%$ the estimated of amplitude uncertainty of the EVN observations.

\section{Summary and conclusion}

1. We obtained total-intensity $1.6 \mathrm{GHz}$ VLBI images of 17 GPS sources for the first time. The majority $(80 \%)$ show a mini-double-lobe radio structure, indicating that they are CSOs or candidates and that their host AGNs could be edgeon to us. This result suggests that there is a high incidence of mini double-lobe sources and CSOs in the GPS source sample.

2. The sources J0323+0534, J1135-0021, J1352+0232, J2058+0540, 2121-014, and 2322-040 with measured redshift are double-lobed with sizes of $<1 \mathrm{kpc}$ and are classified as CSOs.

3. Three sources (J1057+0012, J1600-0037, and 1751+278) are classified as core-jet sources according to their morphologies and flux variability.

4. The $1.6 \mathrm{GHz}$ images of the sources $0914+114,1824+271$, 2121-014, and 2322-040, for which we had observations at $2.3,5.0$, and $8.4 \mathrm{GHz}$, have provided information on their source structure and spectra at the lower frequency, permitting further spectral study in the future.

Acknowledgements. We thank the referee Alvaro Labiano and Nathan de Vries for comments. The European VLBI Network is a joint facility of European, Chinese, South African, and other radio astronomy institutes funded by their national research councils. This research has made use of the NASA/IPAC Extragalatic Database (NED), which is operated by the Jet Propulsion Laboratory, Caltech, under contract with NASA. This work was partly supported by the Natural Science Foundation of China (NSFC).

\section{References}

de Vries, W. H., Barthel, P. D., \& O’Dea, C. P. 1997, A\&A, 321, 105 de Vries, N., Snellen, I. A. G., Schilizzi, R. T., Lehnert, M. D., \& Bremer, M. N. 2007, A\&A, 464, 879

Dallacasa, D. 2004, in Proceedings of the 7th EVN Symposium, ed. R. Bachiller, F. Colomer, et al.

Fanti, C., Fanti, R., Dallacasa, D., et al. 1995, A\&A, 302, 317

Gregory, P. C., \& Condon, J. J. 1991, ApJS, 75, 1011

Griffith, M. R., Wright, A. E., Burke, B. F., \& Ekers, R. D. 1995, ApJS, 97, 347

Labiano, A., Barthel, P. D., O’Dea, C. P., et al. 2007, A\&A, 463, 97

Murgia, M. 2003, PASA, 20, 19

O'Dea, C. P. 1998, PASP, 110, 493

Orienti, M., Dallacasa, D., \& Stanghellini, C. 2007, A\&A, 461, 923

Owsianik, I., \& Conway, J. E. 1998, A\&A, 337, 69

Polatidis, A. G., \& Conway, J. E. 2003, PASA, 20, 69

Snellen, I. A. G., Schilizzi, R. T., Miley, G. K., et al. 2000, MNRAS, 319, 445

Snellen, I. A. G., Lehnert, M. D., Bremer, M. N., \& Schilizzi, R. T. 2002, MNRAS, 337, 981

Stanghellini, C., O’Dea, C. P., Dallacasa, D., et al. 1998, A\&AS, 131, 303

Stanghellini, C., O’Dea, C. P., Dallacasa, D., et al. 2005, A\&A, 443, 891

Tinti, S., Dallacasa, D., de Zotti, G., Celotti, A., \& Stanghellini, C. 2005, A\&A, 432, 31

Tschager, W., Schilizzi, R. T., Röttgering, H. J. A., Snellen, I. A. G., \& Miley, G. K. 2000, A\&A, 360, 887

Xiang, L., Stanghellini, C., Dallacasa, D., \& Haiyan, Z. 2002, A\&A, 385, 768

Xiang, L., Dallacasa, D., Cassaro, P., Jiang, D., \& Reynolds, C. 2005, A\&A, 434, 123

Xiang, L., Reynolds, C., Strom, R. G., \& Dallacasa, D. 2006, A\&A, 454, 729 